\title{
DO THE LINE WIDTHS OF CORONAL EMISSION LINES INCREASE WITH HEIGHT ABOVE THE LIMB?
}

\author{
JAGDEV SINGH \\ Indian Institute of Astrophysics, II Block, Koramangala, Bangalore 560034, India \\ AND \\ TAKAShi SAKURAi AND KiYoshi IChimoto \\ National Astronomical Observatory of Japan, 2-21-1, Ohsawa, Mitaka, Tokyo 181-8588, Japan \\ Received 2005 March 29; accepted 2005 October 27
}

\begin{abstract}
In our earlier studies we obtained off-the-limb spectroscopic observations in a number of forbidden emission lines $([\mathrm{Fe} \mathrm{X}-\mathrm{XIV}])$ to study the physical properties and their temporal variations in steady coronal structures. Short exposure times adopted in those observations permitted us to study the variation in line widths up to about $150^{\prime \prime}$ above the limb. With a view to investigating the variations in the parameters of coronal emission lines up to about $500^{\prime \prime}$, we made raster scans with exposure times that are longer than the earlier exposure times by a factor of about 10 . We find that the FWHM of the [Fe xIV] $5303 \AA$ line decreases up to $300^{\prime \prime} \pm 50^{\prime \prime}$ and then remains more or less the same up to $500^{\prime \prime}$, while that of the $[\mathrm{Fe} \mathrm{x}] 6374 \AA$ line increases up to about $250^{\prime \prime}$ and subsequently remains unchanged. The FWHMs of the $[\mathrm{Fe}$ xI $] 7892 \AA$ and [ Fe XIII] $10747 \AA$ lines show an intermediate behavior. Furthermore, the ratio of the FWHM of 6374 to $5303 \AA$ increases from 0.93 at the limb to 1.18 at $200^{\prime \prime}$ above the limb. The nonvariability in the FWHM of emission lines after about $300^{\prime \prime}$ above the limb in steady coronal structures does not support the prevailing view that the nonthermal velocity increases with height due to either the coronal waves or the high-velocity solar wind. The present results indicate the inadequacy of the earlier coronal loop models. The observed variations in FWHM of the coronal emission lines with height above the limb can be explained by assuming the recent model of coronal loops proposed by Akiyama et al.
\end{abstract}

Subject headings: Sun: corona — techniques: spectroscopic

\section{INTRODUCTION}

The observed intensity oscillations in coronal emission lines and continuum have been interpreted in terms of the existence of coronal waves, which are thought to be responsible for the heating of coronal plasma (Tsubaki 1977; Koutchmy et al. 1983; Pasachoff \& Ladd 1987; Domingo et al. 1995; Singh et al. 1997; Schrijver et al. 1999; Roberts 2000; Sakurai et al. 2002; Minarovjech et al. 2003). The widths of emission lines formed in the transition and coronal regions are greater than those expected from the thermal broadening due to the high temperature of the solar corona (e.g., Boland et al. 1973, 1975; Doschek et al. 1976; Shine et al. 1976; Kjeldseth Moe \& Nicolas 1977; Singh et al. 1982). The observed excess line width has been attributed to the existence of nonthermal broadening due to turbulence or waves in the corona. There have been several attempts to explain the heating of the solar corona and the observed increase of line width with height. Hassler et al. (1990), Doyle et al. (1998), and others have argued that the increase in line width is due to an increase in nonthermal velocity caused by waves. Seely et al. (1997) have questioned the validity of the commonly used assumption of ion temperature being comparable to the electron temperature for the ionization equilibrium. They have argued that if the ion temperature were to increase above the limb, then the increase in temperature could produce the observed line widths without necessarily invoking an increase in the nonthermal velocity.

In our earlier studies we made spectroscopic observations of coronal loops in all four bright coronal emission lines in the visible and near-infrared region of the spectrum, choosing two lines simultaneously (Singh et al. 1999, 2002, 2003a, 2003b). The combination lines were $6374 \AA$ [ $\mathrm{Fe} \mathrm{x}]$ and one of the other three lines, namely, $7892 \AA$ [Fe xI], $10747 \AA$ [ $\mathrm{Fe}$ XIII] , and $5303 \AA$
[Fe XIV]; the infrared lines, 10747 and $10798 \AA$ [Fe XIII], were recorded using the same CCD camera. At that time we were mostly interested in studying the temporal variations, as well as variations in line width with height. We therefore made observations with short exposure times, which permitted us to study line profiles up to a height of about $150^{\prime \prime}$ with a good signal-to-noise ratio from the raster scans extending up to $200^{\prime \prime}$. We found that in almost all the coronal loops the FWHM of the $6374 \AA$ line increased with height irrespective of their shape and type, such as face-on or end-on, radial or nonradial, closed or open, and that the FWHM of the $5303 \AA$ emission line decreased with height above the limb in about $90 \%$ of the coronal loops. The FWHM of the 7892 and $10747 \AA$ lines showed an intermediate behavior with height above the limb (Singh et al. 2003a, 2003b).

The increase in FWHM of the $6374 \AA$ line with height above the limb agreed with most of the measurements made in the EUV lines, probably because both the $6374 \AA$ line and the EUV lines represent plasma at a temperature of about $1 \mathrm{MK}$ (e.g., Doschek et al. 1976; Mariska et al. 1978; Doschek \& Feldman 2000). Recently, Doschek et al. (2001) found that the electron temperature in the coronal hole increases with height above the limb and that the nonthermal motions sometimes increase slightly with height above the limb; when observed, these increases level off at about $120^{\prime \prime}$. We have found an anticorrelation between the temperatures of maximum abundances and gradients of FWHM of the emission lines of the corresponding ions (Singh et al. 2003b). It was surprising to see a decrease in the FWHM of the $5303 \AA$ emission line with height above the limb in addition to this. Our results for the $5303 \AA$ line with height differ from the behavior of other emission lines, especially EUV and 6374 Å lines. Apparently, the reason for such behavior is that the $5303 \AA$ line represents plasma at a relatively high temperature of about $2 \mathrm{MK}$. To 
explain the observed variations of FWHM of the emission lines with height, Singh et al. $(1999,2003 a)$ proposed that mixing of plasma of different temperatures is taking place at larger heights. In such a case the decrease in the FWHM of the $5303 \AA$ line has to stop at some height above the limb. To investigate this we have made spectroscopic observations in all the above-mentioned coronal emission lines up to $500^{\prime \prime}$ above the limb with sufficiently long exposure times. In our earlier letter (Singh et al. 2004) we reported the variation of the intensity ratios of the 5303, 7892, and $10747 \AA$ emission lines to the $6374 \AA$ line as a function of height above the limb and discussed their implications for the coronal loop models and coronal heating. In this paper we report the variation of the FWHM of these lines as a function of height in steady coronal structures and discuss coronal heating mechanisms in view of our new findings.

\section{OBSERVATIONS}

Spectroscopic observations of several coronal regions in the $6374,7892,10747$, and $5303 \AA$ coronal emission lines were obtained sequentially with the $25 \mathrm{~cm}$ coronagraph of the Norikura Solar Observatory on several days during the period of 2003 August-October. The optics of the coronagraph forms the image of the Sun and corona with an image scale of $25^{\prime \prime} \mathrm{mm}^{-1}$, and the $7 \mathrm{~m}$ focal length Littrow-type spectrograph provides a dispersion of $1.17 \AA$ around the $6374 \AA$ line. One of the cameras, which has a $512 \times 512$ format with a pixel size of $27 \mu \mathrm{m}$, was directly mounted at the Littrow focus and was used take spectra in one of the emission lines, mostly the $6374 \AA$ line. The other camera, which has a $1024 \times 1024$ format with a pixel size of $24 \mu \mathrm{m}$, coupled with a $20 \mathrm{~cm}$ aperture with a focal ratio of $\mathrm{f} / 10$ Cassegrain telescope, was located in the beam diffracted from the grating, which overspilled the Littrow mirror of the main spectrograph. Both CCD cameras were manufactured by Photometrics, USA and provided data in 14 byte format.

Earlier we made raster scans with a shorter step size and exposure times of $10-20 \mathrm{~s}$ as mentioned in $\S 1$. For the present study we adopted the parameters of the raster scan and spectrograph so as to obtain a good signal at large heights in the solar corona. We kept the slit width of the spectrograph at $5^{\prime \prime}-6^{\prime \prime}$, since we were interested in recording the faint spectra at large heights. In addition, the spectra were recorded with twice the spectral and spatial resolutions used in earlier studies. This helped us to obtain the spectra close to the limb without saturating the CCD. The raster scans were taken with a step size of $5^{\prime \prime}-20^{\prime \prime}$ as compared to the earlier values of $2^{\prime \prime}-5^{\prime \prime}$ in order to observe a coronal region of $500^{\prime \prime} \times 500^{\prime \prime}$ in a reasonable time. We used an exposure time that is about 10 times the earlier exposure times so as to obtain a good signal-to-noise ratio. The CCD camera provided us with a spatial resolution of about $1^{\prime \prime}$ pixel $^{-1}$ and spectral resolutions of 31.5 , $24.9,124.2$, and $15.8{\mathrm{~m} \AA ~ \text { pixel }^{-1}}^{-1}$ for the $6374,7892,10747$, and $5303 \AA$ emission lines, respectively. A raster scan could be completed in $30-60$ minutes in the case of the 6374 and $5303 \AA$ lines and in 60-100 minutes in the case of the 7892 and $10747 \AA$ lines, depending on the number of steps and the exposure times chosen. The rest of the details of the instrument can be seen in Singh et al. (1999, 2003a).

In this paper we have used the data obtained in the following manner: On 2003 October 26 we made two raster scans of the coronal region near the east limb between the position angles $65^{\circ}$ and $92^{\circ}$ measured from the north pole toward east in the 6374 and $5303 \AA$ coronal emission lines simultaneously. The first scan was made with an exposure time of $50 \mathrm{~s}$. Starting from the limb
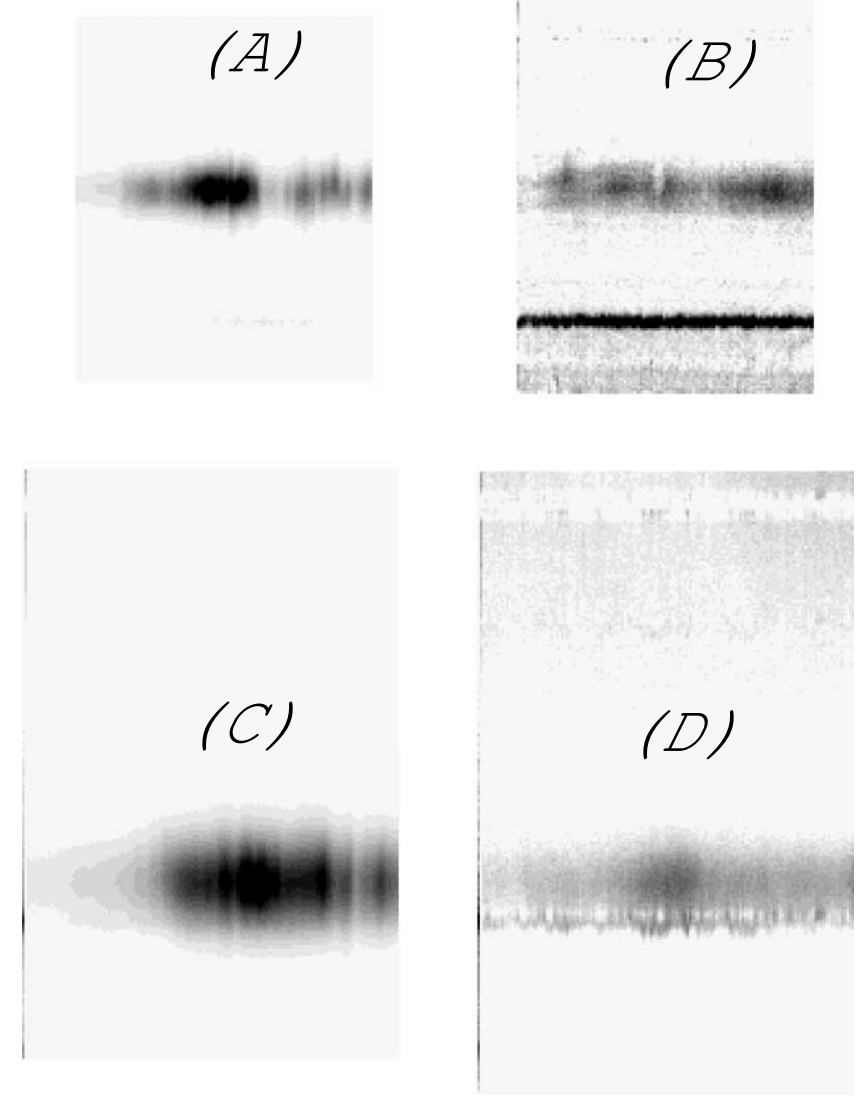

FIG. 1.— $(a, b)[\mathrm{Fe} \mathrm{x}] 6374 \AA \AA$ and $(c, d)$ [Fe xIv] $5303 \AA ̊$ emission-line spectra after correcting for the dark signal, flat-fielding, and scattered light component due to Earth's atmosphere at $20^{\prime \prime}$ and $400^{\prime \prime}$ above the limb obtained on 2003 October 26.

with a step size of $5^{\prime \prime}$, the scan was completed in 50 steps. The slit, corresponding to $6^{\prime \prime} \times 500^{\prime \prime}$, was kept parallel to the limb. Thus, it covered a coronal region of $245^{\prime \prime} \times 500^{\prime \prime}$. The inner corona up to $10^{\prime \prime}$ was covered by the occulting disk of the coronagraph. The second raster scan was made starting from $200^{\prime \prime}$ above the limb and covered a coronal region of $300^{\prime \prime} \times 500^{\prime \prime}$ with a step size of $20^{\prime \prime}$ and exposure time of $200 \mathrm{~s}$. Figure 1 shows four spectra, two in the $6374 \AA$ and two in the $5303 \AA$ emission line obtained at different heights. Figures $1 a$ and $1 b$ show spectra in the $6374 \AA$ line at $20^{\prime \prime}$ and $400^{\prime \prime}$ above the limb, respectively. Similarly, Figures $1 c$ and $1 d$ show spectra in the $5303 \AA$ line at $20^{\prime \prime}$ and $400^{\prime \prime}$. The spectra at $400^{\prime \prime}$ show additional thin black and white lines. These are due to a small remnant signal at the location of photospheric absorption lines caused by the scattered light due to Earth's atmosphere. We have not considered the signal at the locations of absorption lines to compute the Gaussian fit to the emission lines. The spectra at $20^{\prime \prime}$ show the dominance of coronal structures in the lower corona, whereas the spectra at $400^{\prime \prime}$ indicate the dominance of uniform diffused plasma in the higher corona. The spectra at $400^{\prime \prime}$ also indicate that sufficient signal exists at larger heights to determine the line widths accurately.

The observed coronal region overlying the compact sunspot group KKL 22866 was quiet in nature and lasted more than $5 \mathrm{hr}$. No $\mathrm{H} \alpha$ flare occurred in the region before and during the observations. On 2003 October 27 we made a raster scan in the 6374 and $7892 \AA$ coronal emission lines simultaneously over a coronal region of $240^{\prime \prime} \times 500^{\prime \prime}$ size around the position angle $106^{\circ}$ with a 
October 26, 2003

$6374 \AA \quad 5303 \AA$

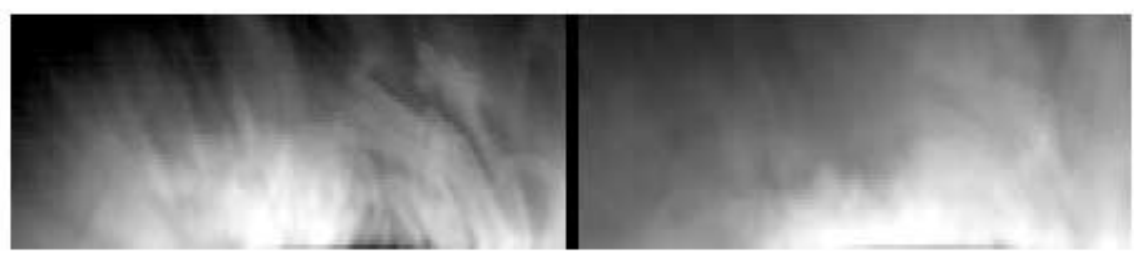

October 27, 2003

$6374 \AA$

$7892 \AA$

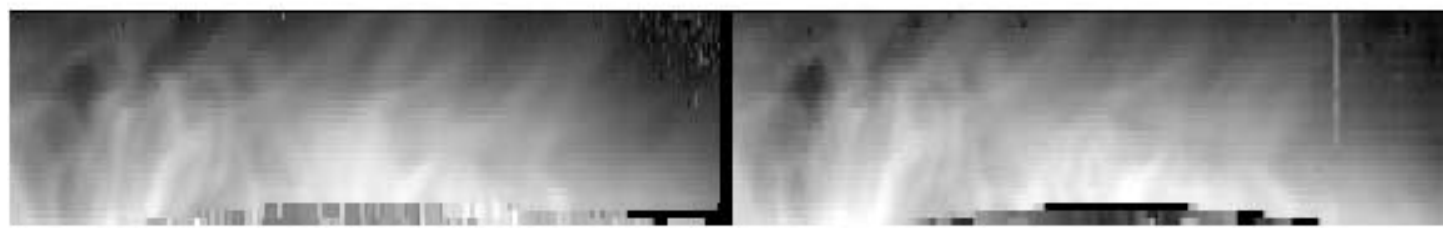

FIG. 2.-Top: Intensity distribution of the $6374 \AA$ (left $)$ and $5303 \AA$ (right) coronal emission lines observed simultaneously in a coronal region of $240^{\prime \prime} \times 500^{\prime \prime}$ size on 2003 October 26. Bottom: Another coronal region of $160^{\prime \prime} \times 500^{\prime \prime}$ size in the 6374 and $7892 \AA$ emission lines observed simultaneously on 2003 October 27.

step size of $6^{\prime \prime}$ and 41 steps. The broadband images of the Sun obtained on October 27 and subsequent days do not show any association with a sunspot group, but magnetograms show moderate magnetic fields at the underlying photosphere. This region was also quiet and did not produce any $\mathrm{H} \alpha$ flare before and during the observing period of about $7 \mathrm{hr}$. In addition, we have used the data obtained in other emission lines, using one emission line at a time, covering several coronal regions observed on different days. Most of the time the sky conditions permitted the observations up to a height of about $300^{\prime \prime}$ only. In these coronal regions, no flare or no large enhancement of the signal was seen during the period of observations.

\section{DATA ANALYSIS}

It is difficult to identify the coronal loops at heights larger than about $200^{\prime \prime}$ above the limb, especially in the intensity images obtained using ground-based coronagraphs. However, with a sufficiently long exposure time it is possible to obtain high-resolution emission-line profiles with good signal-to-noise ratio using a coronagraph located at a good site. We therefore adopted a technique for analyzing the emission-line profiles that differed from our earlier method for identifying the coronal loops and studying the variation of line parameters as a function of height above the limb. First we corrected the spectra for the dark noise, pixel-to-pixel variation in sensitivity, and photospheric scattered light using dark, flat-field, and disk center spectra, respectively, obtained just after the raster scan. The spatial resolution due to the slit width is $6^{\prime \prime}$, whereas each pixel corresponds to a resolution of $1^{\prime \prime}$. To enhance the signal-to-noise ratio, we computed the average of four spectra along the slit for the scan taken with a step size of $20^{\prime \prime}$ only. This resulted in a spatial resolution of $1^{\prime \prime} \times 6^{\prime \prime}$ for the scan taken at the smaller heights with a step size of $5^{\prime \prime}$ and $4^{\prime \prime} \times 6^{\prime \prime}$ for the scan obtained with a step size of $20^{\prime \prime}$ at larger heights. Gaussian fits were then made to the line profiles to compute the peak intensity, line width, and Doppler velocity at each location in the observed coronal region. Figure 2 (top) shows the intensity distribution of a coronal region observed on
2003 October 26 simultaneously in the 6374 and $5303 \AA$ lines. Figure 2 (bottom) shows another coronal region observed on 2003 October 27 simultaneously in the 6374 and $7892 \AA$ lines.

We computed the FWHM from the Gaussian width at each location of the coronal region and corrected it for the instrumental effects as done earlier using the standard relation (Singh et al. 2003a). We have shown typical observed emission-line profiles along with their Gaussian fits in Singh et al. (2003a), indicating excellent quality of the data. The errors in the computed FWHM, given by the standard IDL program, were found to be about $0.001 \AA$ at the lower heights and $0.002 \AA$ around the middle heights (Singh et al. 2003b). They are about $0.005 \AA$ at larger heights around $400^{\prime \prime}$, which is significantly less than the spread in the values of the FWHM. Visual inspection of the fitted Gaussian curves for the low-intensity data showed that at some of the locations, the IDL program used to fit the Gaussian curve generated either a very wide or very narrow Gaussian profile, which did not appear realistic and caused scatter at larger heights in the solar corona. At a given height we did not find any correlation between the FWHM and the intensity of the profile for the faint spectra. Therefore, to exclude the locations of low signal, which are less reliable for further analysis, we selected the spectrum obtained at the largest height in the raster scan and computed the random noise $(\sigma)$ by considering the intensity values of 20 pixels at the continuum level. Then we discarded the values of FWHM for those locations where the line intensity was below the $8 \sigma$ level, which appeared to take care of the unrealistic values of FWHM. At heights up to about $100^{\prime \prime}$ almost all the locations contribute to the plots of FWHM as a function of height as the intensity of the emission line is larger than the $8 \sigma$ level. At heights greater than $100^{\prime \prime}$ the number of locations contributing to the plot decreases as the intensity at some locations become less than $8 \sigma$, but the number still remains significantly large. In Figure 3 we have plotted all the FWHMs thus collected as a function of height above the limb. The plots shown in Figure 3 were obtained from two raster scans: (1) with an exposure time of $50 \mathrm{~s}$ and step size of $5^{\prime \prime}$ between $10^{\prime \prime}$ and $245^{\prime \prime}$ above the limb 

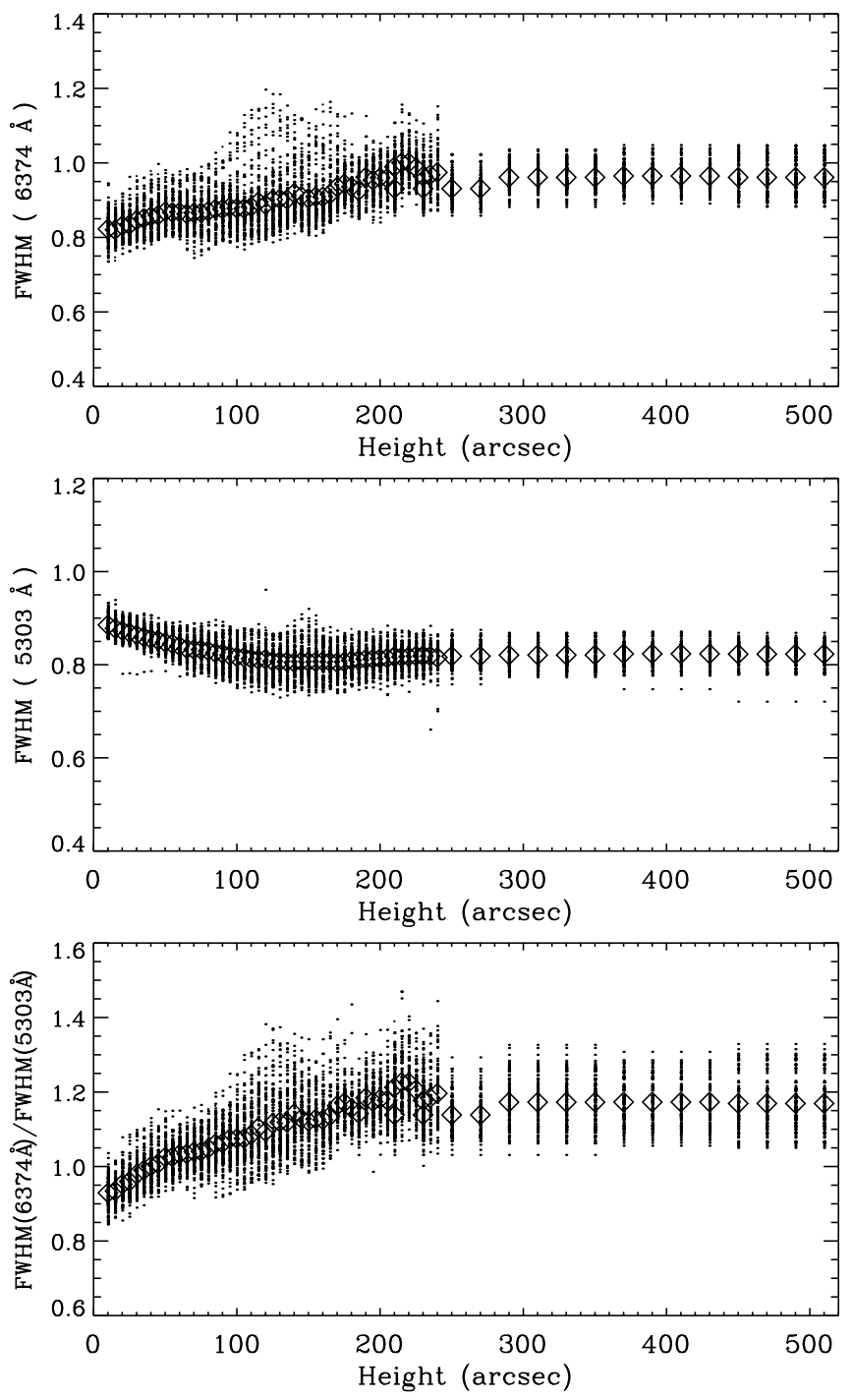

FIG. 3.-Top: FWHM of $6374 \AA$ line as a function of height above the limb in a coronal region observed on 2003 October 26. Middle: FWHM of $5303 \AA$ line simultaneously obtained. Bottom: Ratio of FWHM of 6374 to $5303 \AA$ line as a function of height above the limb. The data from two raster scans obtained with different exposure times and step sizes have been plotted together. The diamonds indicate the mean values of FWHM at each height. The standard deviation in the mean is less than or equal to the height of the symbol.

and (2) with an exposure time of $200 \mathrm{~s}$ and $20^{\prime \prime}$ step size between $200^{\prime \prime}$ and $500^{\prime \prime}$. The difference in the step sizes of the raster scans is clearly seen in the plots.

\section{RESULTS}

\subsection{Variation of FWHM of Emission Lines with Height}

We have plotted the FWHM of the 6374 and $5303 \AA$ lines and their ratio as a function of height above the limb in the three panels of Figure 3 using dots. There are 452 locations with a spatial resolution of $1^{\prime \prime} \times 6^{\prime \prime}$ at each radius up to a height of $245^{\prime \prime}$ and 113 spatial locations with a spatial resolution of $4^{\prime \prime} \times 6^{\prime \prime}$ size at each radius between $200^{\prime \prime}$ and $500^{\prime \prime}$. The data appear in the shape of vertical lines because of the large number of points at each height. The length of the line indicates spread in the FWHM at each radius. The scatter in FWHM at each radius is partly because different coronal loops have different physical characteristics such as temperature, nonthermal velocity, etc. The large scatter in FWHM at smaller heights as compared to that at larger heights may be due to higher spatial resolution at smaller heights. A small coronal region around $120^{\prime \prime}$ height shows large values of FWHM of the $6374 \AA$ line, which might be because of some transient phenomena. It is not clear why the scatter in the FWHM of the $6374 \AA$ line is larger than that in the $5303 \AA$ line. We have also plotted the mean values of FWHM at each height for both emission lines (Fig. 3, diamonds). The standard deviation in the mean values is less than or equal to the height of the symbol used. It may be noted that the standard deviation in the mean values is small compared to the observed variation in the FWHM with height above the limb.

Figure 3 (top) shows that the FWHM of the 6374 A line increases above the limb up to about $200^{\prime \prime}$ as found earlier and then remains unchanged between $200^{\prime \prime}$ and $500^{\prime \prime}$. In addition to this the figure shows that the rate of change of FWHM is not uniform. The middle panel of the figure indicates that the FWHM of $5303 \AA$ line decreases up to about $250^{\prime \prime}$ and then remains more or less unchanged up to $500^{\prime \prime}$. Figure 3 (bottom) indicates that the ratio of FWHM of the $6374 \AA$ to the $5303 \AA$ line (hereafter called FWHM ratio) increases up to about $240^{\prime \prime}$ above the limb. The values of the ratio are 0.93 and 1.18 close to the limb and $200^{\prime \prime}$ away from the limb, respectively. We were able to observe simultaneously in the 6374 and $5303 \AA$ lines up to $500^{\prime \prime}$ only on 2003 October 26.

We have plotted in FWHM all the emission lines as a function of height for seven coronal regions observed on seven different days in Figure 4 to show the variations in other emission lines. One of the coronal regions was observed sequentially in the 7892 and $10747 \AA$ emission lines on the same day. The dates of the observations are indicated in each panel. In the beginning we tried to make raster scans with a step size of $5^{\prime \prime}$ and a long exposure time of $100 \mathrm{~s}$ over a coronal region extending up to $500^{\prime \prime}$ height. However, clouds interrupted after some time, and the raster scan could not be completed. We were able to get spectra up to a height of about $400^{\prime \prime}$. In order to cover the coronal region up to a height of $500^{\prime \prime}$ in a reasonable amount of time, we decided to take one raster scan of a brighter lower coronal region with a shorter exposure time of around $50 \mathrm{~s}$ and another raster scan with a long exposure time and a large step size covering other higher coronal regions. In the process we could get better signal-tonoise ratio at larger heights.

The observations obtained on September 4 and 9 indicate that the FWHM of the $5303 \AA$ line continued to decrease with height up to $300^{\prime \prime}$ and $400^{\prime \prime}$, respectively, whereas in the coronal region observed on September 7, the FWHM of the $5303 \AA$ line decreased up to $300^{\prime \prime}$ and then remained the same up to $360^{\prime \prime}$ (Fig. 4). The FWHM of the $5303 \AA$ emission line cannot continually decrease with height because the FWHM will have a minimum value corresponding to the thermal broadening. It has to reach a minimum value at some height, which may remain the same at larger heights or may begin to increase with height. The coronal region observed on October 26 indicates that the FWHM reaches a minimum value of about $0.82 \AA$ at a height of $200^{\prime \prime}$ (Fig. 3) and then does not vary with height. The height at which the FWHM of the $5303 \AA$ line becomes a minimum and does not decrease further may depend on the physical characteristics, such as magnetic field, density, temperature, etc., of the coronal region. More observations at larger heights extending up to $600^{\prime \prime}$ or more are needed.

The coronal regions observed in the $6374 \AA$ emission line on August 20 and October 8 clearly show that the mean FWHM of the line increases with height up to about $200^{\prime \prime}$ and then remains more or less the same at larger heights (Fig. 4), as in the case of those observed on October 26. The coronal region observed on October 9 indicates that the mean FWHM of $7892 \AA$ emission 

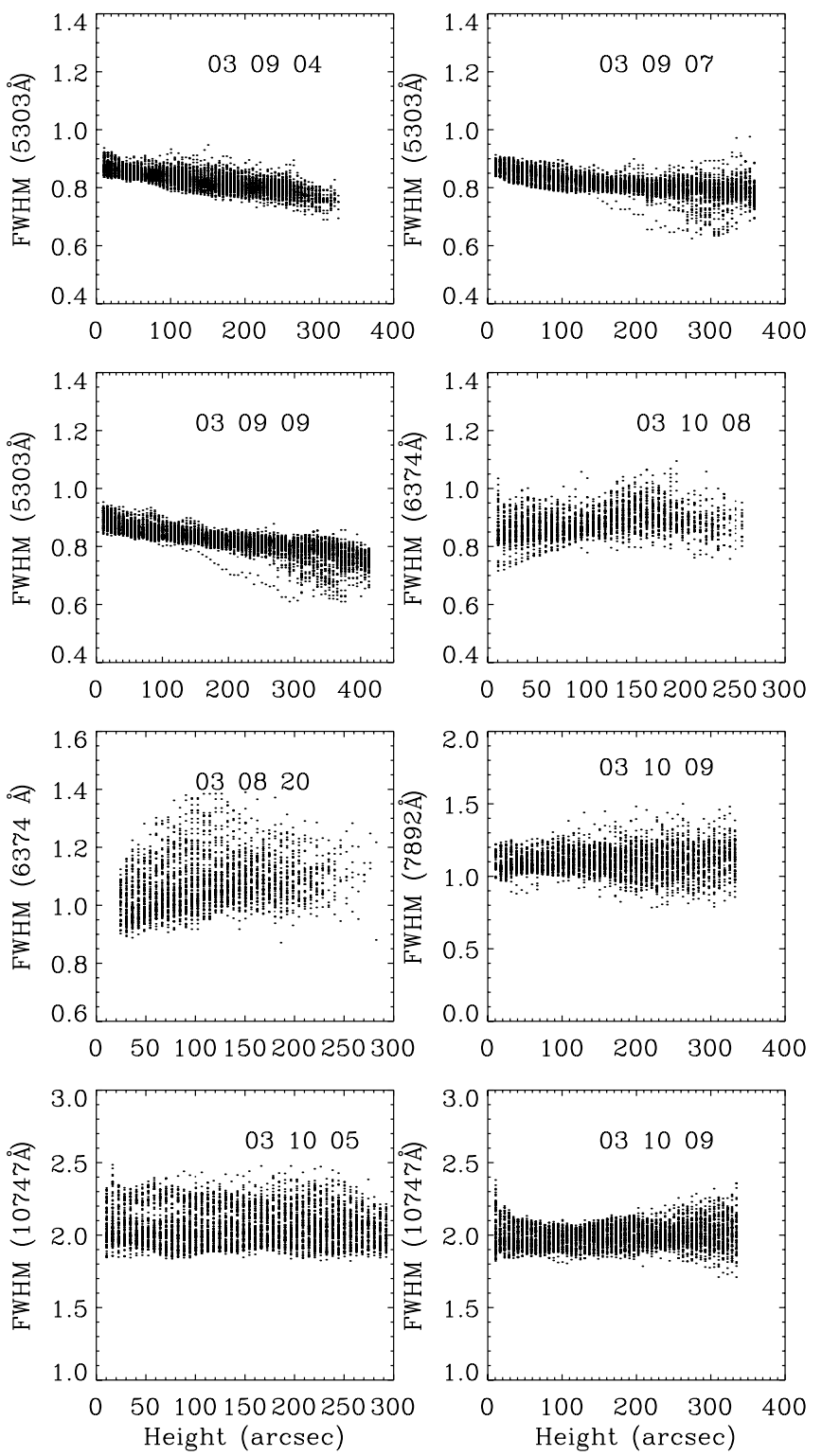

FIG. 4.-FWHM of the 5303, 6374, 7892, and $10747 \AA$ lines as a function of height above the limb in different coronal regions observed during the period of 2003 August-October. The date of observation (yy $\mathrm{mm} \mathrm{dd}$ ) is indicated in each panel, and the emission line used is written along the $y$-axis.

lines increases marginally up to about $100^{\prime \prime}$ and then remains more or less the same at larger heights. Figure 4 shows that the mean FWHM of the $10747 \AA$ line also varies marginally with height above the limb for the coronal regions observed on October 5 and 9 .

\subsection{Distribution of FWHM of Lines at a Given Height}

We have shown the distribution of the FWHM of 6374 and $5303 \AA$ emission lines observed on 2003 October 26 at two representative heights of $50^{\prime \prime}$ and $300^{\prime \prime}$ above the limb in Figure 5. The distributions of FWHM of both emission lines have skewness with tails extending at higher values of FWHM. The distribution of FWHM of the $6374 \AA$ line at $50^{\prime \prime}$ and $300^{\prime \prime}$ peaks around 0.83 and $0.94 \AA$, respectively, while that of the $5303 \AA$ line peaks around 0.87 and $0.82 \AA$, respectively. It is clear that the changes in the mean values of FWHM of the 6374 and $5303 \AA$ lines with height are not because of any change in the shape
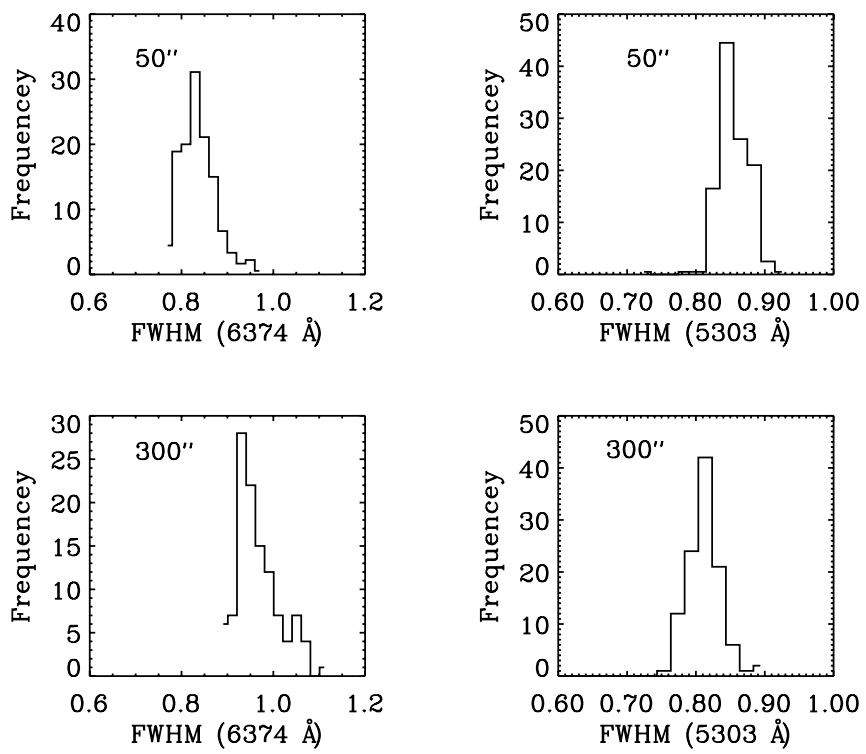

FIG. 5.-Distribution of FWHM of the 6374 and $5303 \AA$ emission lines at the two heights, namely, $50^{\prime \prime}$ and $300^{\prime \prime}$, for the observations made on 2003 October 26.

of the distribution; there is real change with height in the mean value of FWHM about which the observed values are distributed. The distribution plots confirm the findings that the FWHM of the $6374 \AA$ line increases with height and that of the $5303 \AA$ line decreases with height above the limb.

\subsection{Correlation between FWHMs of Two Emission Lines at a Height}

Figure 6 shows plots of FWHM of the $5303 \AA$ versus that of the $6374 \AA$ emission line at the four different heights, namely, $90^{\prime \prime}, 170^{\prime \prime}, 250^{\prime \prime}$, and $350^{\prime \prime}$, for the data obtained on 2003 October 26. The figure also shows plots of FWHM of the $7892 \AA$ versus that of the $6374 \AA$ line at $20^{\prime \prime}$ and $150^{\prime \prime}$ for the data taken on 2003 October 27. The solid line in each panel indicates the linear fit to the data set, and the values of correlation coefficient and height for each data set are given in each panel. There is no correlation between the FWHM of the 5303 and $6374 \AA$ lines at heights less than $80^{\prime \prime}$ or so. The plots at $90^{\prime \prime}$ and $170^{\prime \prime}$ height show a large spread, but there is a significant indication of a positive correlation. The correlation coefficients derived are 0.42 and 0.44 , respectively. The FWHM of the 6374 and $5303 \AA$ lines appears to be anticorrelated at the height of $250^{\prime \prime}$, with the correlation coefficients being -0.32 . There is again no correlation between the FWHM of the two lines at larger heights as seen from the plot at $350^{\prime \prime}$ in Figure 6. It is difficult to explain such behavior. Probably the FWHMs of the two lines are uncorrelated at smaller heights because of the superposition of a number of coronal loops in the line of sight and small spatial differences in the locations of the 6374 and $5303 \AA$ line loops. At the middle heights $\left(100^{\prime \prime}-200^{\prime \prime}\right)$, superposition of loops decreases and coronal loops become prominent and cospatial due to broadening of loops; this probably leads to a positive correlation between the FWHMs of the 6374 and $5303 \AA$ lines at these heights. At larger heights, the diffused plasma is dominant and there are no systematic variations in FWHM of the emission lines with height. The scatter in the plots may be mainly due to small-scale inhomogeneities in the solar corona.

The two bottom panels of Figure 6 indicate a significant correlation between the FWHMs of the 7892 and $6374 \AA$ emission lines. The computed correlation coefficients are 0.45 and 0.53 at 

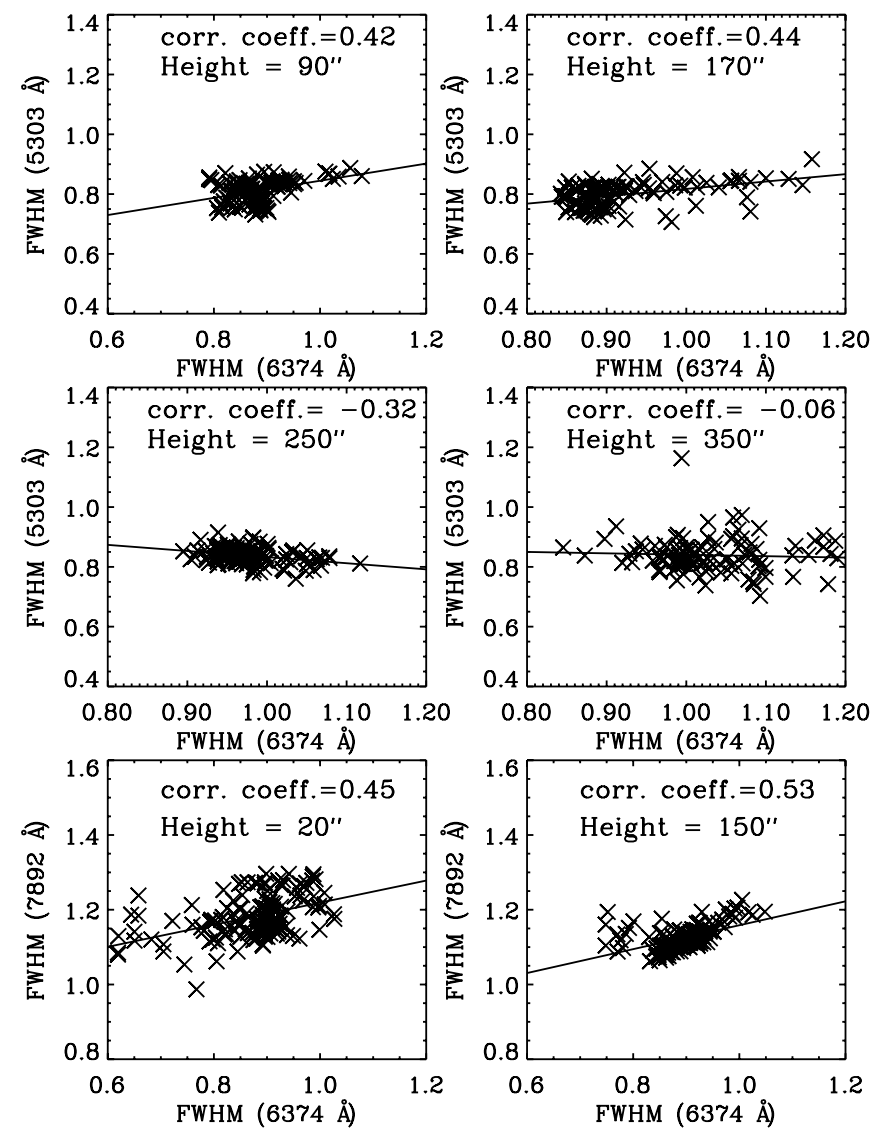

FIG. 6.-Top and middle: FWHM of the $5303 \AA$ line against the FWHM of the $6374 \AA$ emission line at the four heights, namely, $90^{\prime \prime}, 170^{\prime \prime}, 250^{\prime \prime}$, and $350^{\prime \prime}$, for the observations made on 2003 October 26. Bottom: FWHM of the $7892 \AA$ vs. FWHM of the $6374 \AA$ line at $20^{\prime \prime}$ and $150^{\prime \prime}$ for the observations made on 2003 October 27

the heights of $20^{\prime \prime}$ and $150^{\prime \prime}$, respectively. The correlation reaches a maximum of $\sim 0.7$ around a height of $100^{\prime \prime}$. The FWHM of coronal emission lines, as considered generally, represents a constant thermal component and a varying nonthermal component. Therefore, a good correlation between the FWHMs of the 7892 and $6374 \AA$ lines and a significant positive correlation between the FWHMs of the 5303 and $6374 \AA$ lines in the middle corona indicate a correlation between the nonthermal velocities in the coronal structures representing different temperature plasma in the range of $(1-1.8) \times 10^{6} \mathrm{~K}$. These results agree with the high degree of correlation among the nonthermal motions in the transition regions at temperatures ranging from $3 \times 10^{4}$ to $2.5 \times 10^{5}$ found by Akiyama et al. (2005). The difference in the degree of correlation is probably because the present observations represent high-temperature plasma as compared to the transition region plasma.

\subsection{Comparison with Isolated Individual Coronal Loops}

To compare the average variations of FWHM with height above the limb with those in a well-defined individual coronal loop, we show in Figure 7 typical examples of coronal regions observed in the $6374 \AA$ line and one of the other three emission lines simultaneously on three different days with short exposure times up to a height of $200^{\prime \prime}$. The top two panels of Figure 7 show the intensity distribution in the 6374 and $7892 \AA$ emission lines, respectively, in a coronal region observed simultaneously. The middle panels show the intensity distribution in the 6374 and
$10747 \AA$ lines in a different coronal region, and the bottom panels show that in the 6374 and $5303 \AA$ emission lines. To study variations of FWHM with height along a coronal loop, we displayed on a monitor screen the $6374 \AA$ line image of a coronal region and chose locations along a coronal loop with the help of the cursor. These locations were automatically selected on the other images by a computer program. Typical examples of coronal loops chosen from the $6374 \AA$ line image and automatically selected from the 7892, 10747, and $5303 \AA$ emission lines are shown in Figure 7 by plus signs. The variations of FWHM of the emission lines as a function of height in these well-defined coronal loops are shown in Figure 8. The figure shows a smooth variation in FWHM of the emission lines along the loops. Linear fits to the data of well-defined loops show that the FWHM of the $6374 \AA$ line increases at a faster rate than those of the 7892 and $10747 \AA$ lines. It is found that in about $57 \%$ of the loops the FWHM of the $10747 \AA$ line increases with height and in the remaining loops it decreases with height (Singh et al. 2003a). This is surprising, since we expect a larger increase in the FWHM of the 7892 and $10747 \AA ̊$ lines with height than that of the $6374 \AA$ line if this increase is due to an increase in temperature or nonthermal velocity with height in coronal loops. The decrease in FWHM of the $5303 \AA$ line with height as seen in the bottom right panel indicates a decrease in temperature or nonthermal velocity or both with height in coronal loops. It can be noted that the variations in the FWHM of the emission lines in the coronal loops have been studied only up to a height of about $150^{\prime \prime}$. The results of this study agree with the average variations in the FWHM of emission lines in the lower corona up to $150^{\prime \prime}$. Therefore, it may be safe to conclude that the observed variations in the FWHM with height are the general characteristics of the solar corona.

\section{DISCUSSION}

We find that the FWHM of the $6374 \AA$ line increases significantly only up to about $200^{\prime \prime}$ and the FWHM of the $5303 \AA$ line first decreases up to $300^{\prime \prime} \pm 50^{\prime \prime}$ and then remains more or less unchanged with height above the limb. The variations in the FWHM of the 7892 and $10747 \AA$ emission lines show an intermediate behavior. We find that in almost all the coronal loops the FWHM of the $6374 \AA$ line increased with height irrespective of their shape and type, and the FWHM of the $5303 \AA$ emission line decreased with height above the limb in about $90 \%$ of the coronal loops (Singh et al. 2003a). The behavior of FWHM of these lines with height is found to be nearly the same when all the coronal loops and diffused plasma were considered together. The average FWHM of the $7892 \AA$ line increases by a small amount up to a height of about $150^{\prime \prime}$ and then remains more or less the same. The average FWHM of the $10747 \AA$ line shows marginal variations with height above the limb.

It has been shown (e.g., Hollweg 1973; Esser et al. 1987) that hydromagnetic waves will produce significant broadening of high-temperature emission lines observed above the limb if the waves have velocity amplitudes of more than $10 \mathrm{~km} \mathrm{~s}^{-1}$ at the coronal base. In particular, if the waves are undamped, the wave velocity amplitude, and hence the observed line width, should increase sharply above the limb. Since near the coronal base the density falls off exponentially with increasing radial distance, the wave velocity amplitude is expected to increase exponentially with radius, causing the emission line widths to increase rapidly (Hassler et al. 1990). In order to isolate line broadening due to hydromagnetic waves, it is necessary to observe the line widths of high-temperature coronal lines (temperature of formation $>10^{6} \mathrm{~K}$ ) above the limb where broadening of this nature 


\section{September 20, 1998}

\section{$6374 \AA$} $7892 \AA$

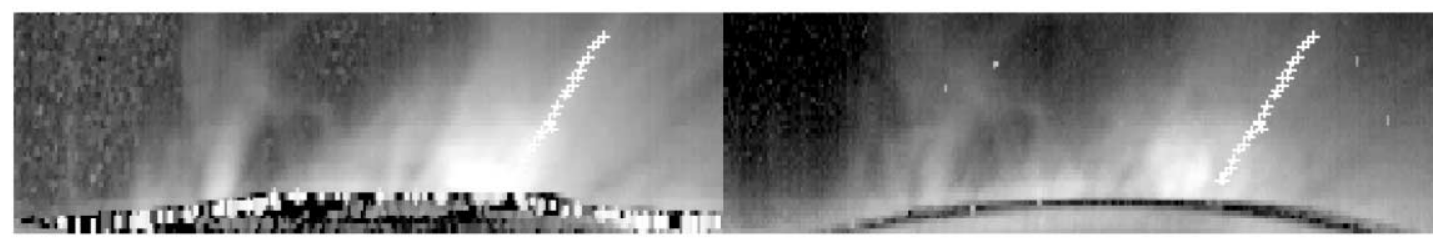

September 10, 1998

$6374 \AA$

$10747 \AA$

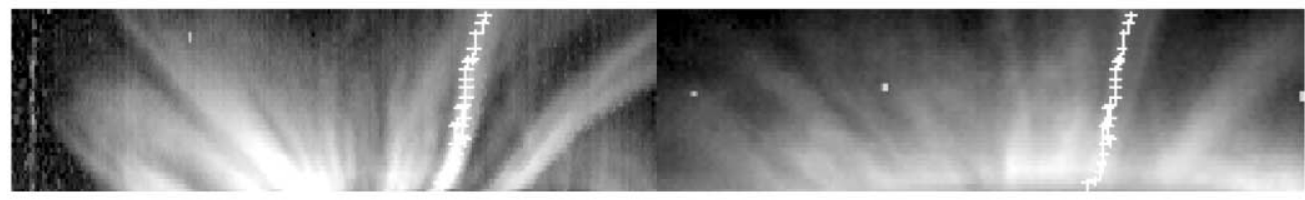

September 19, 1997

$6374 \AA$

$5303 \AA$

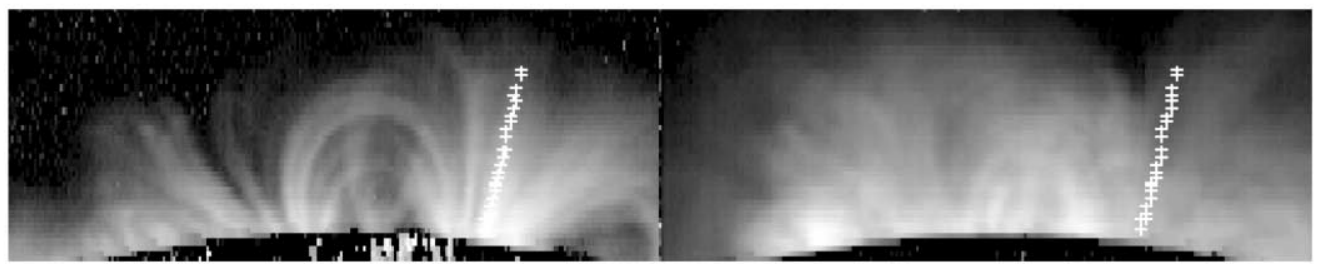

Fig. 7.- Intensity distribution in coronal regions in $6374,7892,10747$, and $5303 \AA$ emission lines. Typical coronal loops in each emission line are shown by plus signs.

becomes dominant (Hassler et al. 1990). The increase in FWHM of the $6374 \AA$ line supports the existence of hydromagnetic waves in the corona as suggested by Hassler et al. (1990) and Doyle et al. (1998).

The waves, which cause an increase in FWHM of the $6374 \AA$ line with height above the limb, are also expected to cause a corresponding increase in line widths of the 7892, 10747, and $5303 \AA$ emission lines. However, the increase in FWHM of the $7892 \AA$ line with height is much smaller than that expected from the observed increase in FWHM of the $6374 \AA$ line, and the increase in FWHM of the $10747 \AA$ line is only marginal. In addition to these, the decrease in FWHM of the $5303 \AA$ line with height contradicts the scenario proposed by Hassler et al. (1990) and others. The line widths of all four observed emission lines reach near-constant values at heights between $200^{\prime \prime}$ and $300^{\prime \prime}$ and thereafter remain more or less unchanged up to $500^{\prime \prime}$ above the limb. It can be argued that the hydromagnetic waves, which cause the increase in FWHM of some of the emission lines up to about $250^{\prime \prime}$, may be getting damped beyond this. If the hydromagnetic waves exist in the lower corona, then why does the line width of the $5303 \AA$ line decrease with height above the limb? In addition, the presence of the solar wind at larger heights $\left(>300^{\prime \prime}\right)$ is expected to cause an increase in the FWHM of coronal emission lines with height, but we find no variation in line widths between $300^{\prime \prime}$ and $500^{\prime \prime}$. The behavior of all the emission lines put together does not favor the existence of hydromagnetic waves in steady coronal structures. Furthermore, the solutions of hydrodynamic equations for the coronal loops predict a steep temperature rise from the loop footpoint through the transition region and a small but measurable temperature increase from the top of the transition region to the loop apex (Winebarger et al. 2003). The increase in temperature along a loop is expected to cause an increase in the FWHM of the coronal emission lines with height. The observed increase in FWHM of the $6374 \AA$ line with height above the limb is consistent with this scenario, while the decrease in FWHM of the $5303 \AA$ line with height is not. The variations in the FWHM of the four emission lines observed are very complex, and hence it is difficult to interpret the findings in simple terms of monotonic increase or decrease in the coronal temperature or nonthermal velocity with height above the limb.

Doschek et al. (2001) showed that the line broadening above the limb is a variable feature, depending on the coronal conditions at the time of observations. Our observations of coronal emission line profiles spread over a period of $7 \mathrm{yr}$ also indicate that the magnitude of the rate of FWHM variation with height above the limb in different coronal structures is different (Singh et al. 1999, 2003a, 2003b). However, the general pattern in the variation of FWHM with height in coronal emission lines remains the same; e.g., most of the coronal loops observed show a decrease in the FWHM of the $5303 \AA$ line with height and an 

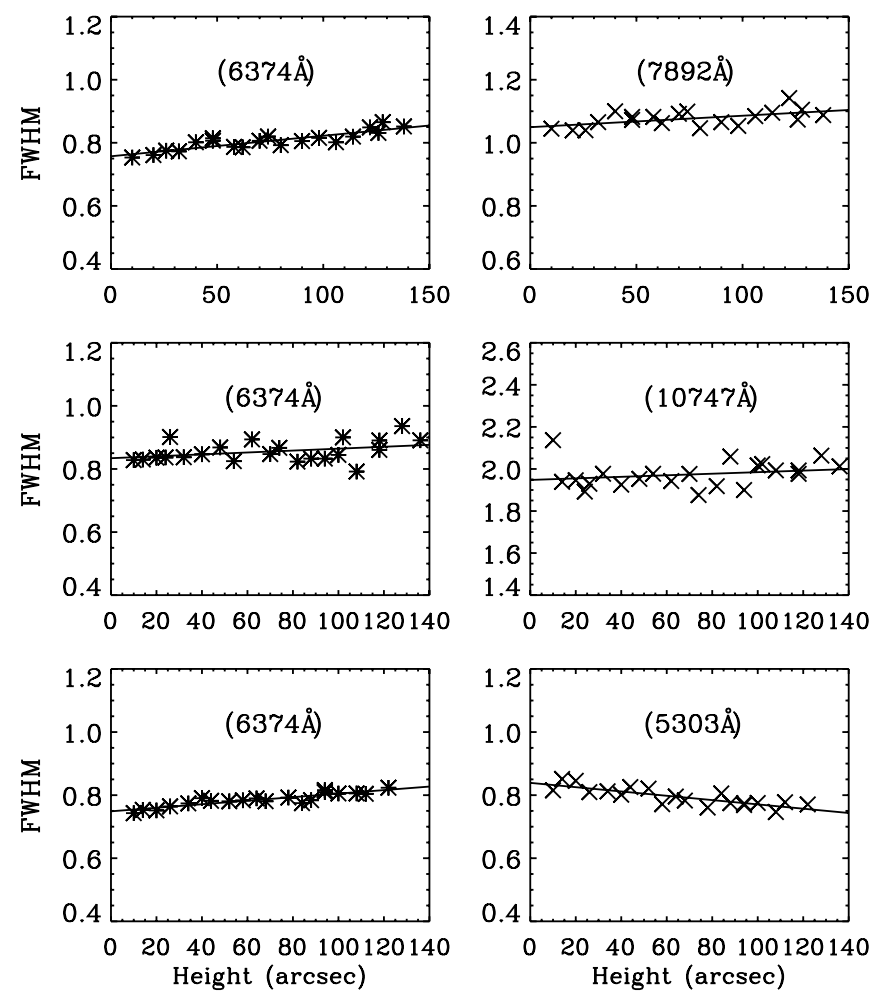

FIG. 8.-FWHM of $6374,7892,10747$, and $5303 \AA$ emission lines as a function of height above the limb for the coronal loops shown in Fig. 7. The linear fit to the respective data set has also been plotted.

increase in the FWHM of the $6374 \AA$ line with height irrespective of the shape of the loop. The 7892 and $10747 \AA$ lines show an intermediate trend. The differences in rates of variation of the FWHM in different coronal structures may be due to differences in the underlying photospheric magnetic field configuration, density, temperature, and state of evolution of the structure.

Recently, Akiyama et al. (2005) analyzed the relationship between nonthermal velocities derived from the spectral lines of ions formed at different temperatures in the solar lower transition region using spectra from Solar Ultraviolet Measurements of Emitted Radiation (SUMER). They found a good correlation between nonthermal velocities for a number of pairs of simultaneously observed emission lines. They therefore suggested that there is a close connection between the plasma at different temperatures over a temperature regime of $3.2 \times 10^{4}$ to $2.5 \times 10^{5} \mathrm{~K}$ and that this connection might expand into the dynamic realm. The measurements of the widths of the 6374 and $5303 \AA$ emission lines in the coronal loops suggest a similar close relation between plasma at different temperatures in the regime of $1 \times 10^{6}$ to $1.8 \times 10^{6} \mathrm{~K}$ (Singh et al. 2003a). Variations in FWHM of both emission lines with height were found to be almost the same in the adjacent and similar coronal loops; only the intensity ratio varied from loop to loop.

A similar trend in the variation of the FWHM of emission lines has been found in the spatially resolved coronal loops as shown in Figures 7 and 8, as well as in the coronal region considered as a whole. Therefore, the variations found in the FWHM of emission lines with height above the limb are of the same nature all over the corona, including coronal loops. It is possible that different temperature regions are produced by unresolved, physically distinct isothermal loops with sizes substantially less than $1^{\prime \prime}$. In addition, it is possible that different temperature plasma loops behave differently. Then it is difficult to find a physical mechanism that will cause the FWHM of the $6374 \AA$ line to increase steeply compared to that of the $7892 \AA$ line when both ions have a small difference in the temperature of their maximum abundances. Actually, the FWHM of the $7892 \AA$ line is expected to increase steeply, as a similar increase in temperature or nonthermal velocity will increase the line width proportional to its wavelength. Furthermore, there exists an anticorrelation between the gradient of FWHM with height and the temperature of maximum abundances of the ions (Singh et al. 2003b). It is rather difficult to explain the observed variations in line widths of coronal emission lines in terms of wave heating models and probably dynamical heating models as well if coronal loops are assumed to be single-temperature isothermal thin loops where magnetic pressure is high compared to the gas pressure. It is not clear how the dynamical heating of loops will lead to a decrease in the FWHM of the $5303 \AA$ emission line with height.

It is very simple to explain the observed variations in FWHM of all the lines in terms of the recently proposed model by Akiyama et al. (2005). They propose that although the different temperature plasmas that exist in discrete locations in the same loop are not spatially resolved, there is a physical relationship between each plasma region because all the plasma is confined to a single loop. They conclude that the different temperature regions are not magnetically isolated from each other and can therefore be physically connected through thermal conduction. In this scenario, we can say that contributions to the $5303 \AA$ emission come from the relatively hot plasma and those to the $6374 \AA$ line from the relatively cold plasma closer to the solar limb. Thus, the FWHM of the $6374 \AA$ line is smaller and that of the $5303 \AA$ line is larger at smaller heights. However, at intermediate $\left(100^{\prime \prime}-200^{\prime \prime}\right)$ heights, due to thermal conduction between the different temperature plasmas, the temperature of hotter plasma decreases and that of cooler plasma increases. In this process nonthermal velocities may also share part of their energy. This leads to a decrease in line width of the $5303 \AA$ line and an increase in that of the $6374 \AA$ emission line with height above the limb. At larger heights $\left(300^{\prime \prime}-\right.$ $500^{\prime \prime}$ ), all the plasmas reach a state of more or less uniform temperature and, probably, nonthermal velocity by conduction in different temperature plasmas. Therefore, the FWHM of emission lines does not vary with height in the $300^{\prime \prime}-500^{\prime \prime}$ range. If steady coronal loops do not extend this height, diffused plasma around the coronal loops shows this behavior of line widths of emission lines. The plasmas representing the 7892 and $10747 \AA$ emission lines are already at an intermediate temperature near the limb and close to a uniform temperature at larger heights; therefore, these two emission lines show marginal variations in the line widths.

In conclusion, our observations do not support the prevailing view that the nonthermal velocity increases with height due to coronal waves in steady coronal structures. The new findings about the variation of FWHM of four coronal emission lines at larger heights imply certain constraints on the models of coronal loops, the location of the origin of the solar wind, and the coronal heating. More observations in the EUV and X-ray lines with high spectral resolution are needed to understand the dynamics of steady coronal loops.

We thank the referee for valuable comments, which have improved the quality of the paper to a large extent. J. Singh thanks the members of the Norikura Observatory for the help they extended to him during the observations and A. V. Raveendran for useful comments. 
Akiyama, S., Doschek, G. A., \& Mariska, J. T. 2005, ApJ, 623, 540

Boland, B. C., Engstrom, S. F. T., Jones, B. B., \& Wilson, R. 1973, A\&A, 22, 161

Boland, B. C., et al. 1975, MNRAS, 171, 697

Domingo, V., Fleck, B., \& Poland, A. I. 1995, Sol. Phys., 162, 1

Doschek, G. A., \& Feldman, U. 2000, ApJ, 529, 599

Doschek, G. A., Feldman, U., Laming, J. M., Schuhle, U., \& Wilhelm, K. 2001, ApJ, 546, 559

Doschek, G. A., Feldman, U., VanHoosier, M. E., \& Bartoe, J. D. F. 1976, ApJS, 31, 417

Doyle, J. G., Banerjee, D., \& Perez, M. E. 1998, Sol. Phys., 181, 91

Esser, R., Holzer, T. E., \& Leer, E. 1987, J. Geophys. Res., 92, 13377

Hassler, D. M., Rottman, G. J., Shoub, E. C., \& Holzer, T. E. 1990, ApJ, 348, L77

Hollweg, J. V. 1973, ApJ, 181, 547

Kjeldseth Moe, O., \& Nicolas, K. R. 1977, ApJ, 211, 579

Koutchmy, S., Zhugzda, Y. D., \& Locans, V. 1983, A\&A, 120, 185

Mariska, J. T., Feldman, U., \& Doschek, G. A. 1978, ApJ, 226, 698

Minarovjech, M., Rusin, V., Rybansky, M., Sakurai, T., \& Ichimoto, K. 2003, Sol. Phys., 213, 269
REFERENCES

Pasachoff, J. M., \& Ladd, E. F. 1987, Sol. Phys., 109, 365

Roberts, B. 2000, Sol. Phys., 193, 139

Sakurai, T., Ichimoto, I., Raju, K. P., \& Singh, J. 2002, Sol. Phys., 209, 265

Schrijver, C. J., et al. 1999, Sol. Phys., 187, 261

Seely, J. F., Feldman, U., Schuhle, U., Wilhelm, K., Curdt, W., \& Lemaire, P. 1997, ApJ, 484, L87

Shine, R. A., Roussel-Dupre, D., Bruner, E. C., Jr., Chipman, E. G., Lites, B. W., Rottman, G. J., Athay, R. G., \& White, O. R. 1976, ApJ, 210, L107

Singh, J., Bappu, M. K. V., \& Saxena, A. K. 1982, J. Astrophys. Astron., 3, 249

Singh, J., Ichimoto, K., Imai, H., Sakurai, T., \& Takeda, A. 1999, PASJ, 51, 269

Singh, J., Ichimoto, K., Sakurai, T., \& Muneer, S. 2003a, ApJ, 585, 516

Singh, J., Sakurai, T., Ichimoto, K., \& Muneer, S. 2003b, Sol. Phys., 212, 343

Singh, J., Sakurai, T., Ichimoto, K., Suematsu, Y., \& Takeda, A. 2002, PASJ, 54,793

Singh, J., Sakurai, T., Ichimoto, K., \& Watanabe, T. 2004, ApJ, 617, L81

Singh, J., et al. 1997, Sol. Phys., 170, 235

Tsubaki, T. 1977, Sol. Phys., 51, 121

Winebarger, A. R., Warren, H. P., \& Seaton, D. B. 2003, ApJ, 593, 1164 